\title{
Magnetization Reversal Mechanisms in Colloidal Dispersions of Magnetite Particles
}

\author{
J. M. González and M. I. Montero \\ Instituto de Ciencia de Materiales de Madrid-CSIC. Cantoblanco, 28049 Madrid. Spain \\ J. A. López-Pérez, J. Mira, M. A. López-Quintela and J. Rivas \\ Facultade de Fisica. Universidade de Santiago de Compostela. \\ Campus Universitario. 15706 Santiago de Compostela. Spain \\ $X$. Batllé and A. Labarta \\ Facultat de Fisica. Universitat de Barcelona. Diagonal 647.08028 Barcelona. Spain.
}

\begin{abstract}
We have studied the structural and magnetic properties of colloids consisting of different concentrations of magnetite particles dispersed in kerosene. Our data for the temperature dependence of the low field magnetization and the saturation coercive force of the samples evidenced the occurrence, in different temperature ranges, of Néel- and Brown-type relaxation processes whose characteristics were largely influenced by strong dipolar interparticle interactions.
\end{abstract}

Index terms- Coercivity, colloid, relaxation, dipolar interactions

\section{INTRODUCTION.}

S table colloidal suspensions of magnetically ordered particles ("ferromagnetic liquids") have evidenced to be the most performing materials in high rotating speed seals [1]. They have also been used as magnetic lubricants, in stepping motors and loudspeaker coils (to provide magnetic damping) and in different energy conversion devices [1]. Apart from the relevance of their practical uses, these colloids are also interesting from the point of view of the study of the vast phenomenology of superparamagnetism under controlled dipolar interparticle interactions [2,3].

In the present work we report on the temperature dependence of the low field magnetization and the coercive force of a series of colloids having different magnetic phase concentrations and prepared from the same batch of magnetite nanoparticles.

\section{PREPARATION OF SAMPLES AND EXPERIMENTAL TECHNIQUES.}

A peptization method was used to prepare the magnetic particles [4] (a solution containing $\mathrm{Fe}^{+2}$ and $\mathrm{Fe}^{+3}$ salts in the $1: 2$ molar ratio was added over an ammonia one). After precipitation, the obtained nanoparticles were covered with oleic acid and washed several times with large amounts of water and ketone. Finally the particles were dispersed in kerosene to obtain a stable magnetic colloid. Three different samples $A, B$ and $C$ having magnetite particle volume percentages of $0.2,0.8$, and 4.5 , respectively, were prepared.

Manuscript received October 1,1997

J.M. Gonzalez +34-1-3349052, fax +34-1-3720623

immgf4a@fresno.esic.es.

http: $/$ www.icmm.csic.es/jmgonzalez/persi.htinl
The structural characterization of the magnetite particles was carried out by using X-rays diffraction (XRD) whereas the study of their average dimensions was completed by means of transmission electron microscopy (TEM). The magnetic measurements were performed in a SQUID magnetometer.

\section{EXPERIMENTAL RESULTS.}

The diffraction pattern of the particles shows the characteristic peaks of the magnetite. A crystallite size of 11 nm was obtained by considering the Scherrer formula [5] to analyze the width of the most intense peak. This result was in good agreement with the evaluation of the same quantity carried out by means of TEM which yield an average particle size of $8 \pm 3 \mathrm{~nm}$. On the basis of the TEM result we have evaluated the nominal average interparticle distance which was of $46 \mathrm{~nm}$ ( 5.75 average diameters), $24 \mathrm{~nm}$ ( 3 average diameters) and $10 \mathrm{~nm}$ ( 1.25 average diameters) in the cases of the $A, B$ and $C$ samples, respectively.

As for the magnetic properties of the samples, Figs. 1a), 1b) and 1c) show the results obtained in the three colloids for the temperature dependence of the low field $(5 \mathrm{Oe})$ magnetization measured, by heating up the samples after cooling them down to $5 \mathrm{~K}$, under zero applied field (ZFC curves) and under an applied field of $5 \mathrm{Oe}$ ( $\mathrm{FC}$ curves). In the ZFC curve measured in sample $A$. (that having the lower concentration of magnetite particles) two maxima, taking place at ca. $100 \mathrm{~K}$ and $230 \mathrm{~K}$, respectively, can be observed. The high temperature maximum is also observable in the corresponding FC curve. Sample B shows a behavior similar to that described for sample $A$, with the only remarkable differences of the temperature of the first maximum (which now is observed at ca. $140 \mathrm{~K}$ ) and of the larger width of the second maximum (onseting at $175 \mathrm{~K}$, peaking at ca. $230 \mathrm{~K}$ and showing some structure). Finally, in the more concentrated sample $(\mathrm{C})$ it is possible to observe that the low temperature maximum overlaps with a new one developing in the temperature range going from 175 up to $220 \mathrm{~K}$. Similarly to the case of the other samples a third maximum is measured at ca. $230 \mathrm{~K}$.

In Figs. 2a), 2b) and 2c) we present our results for the temperature variation of the coercive force, $\mathrm{H}_{0}(\mathrm{~T})$ (measured after saturating the samples in a 5 kOe field). Our coercivity data were only slightly sensitive to the concentration of 

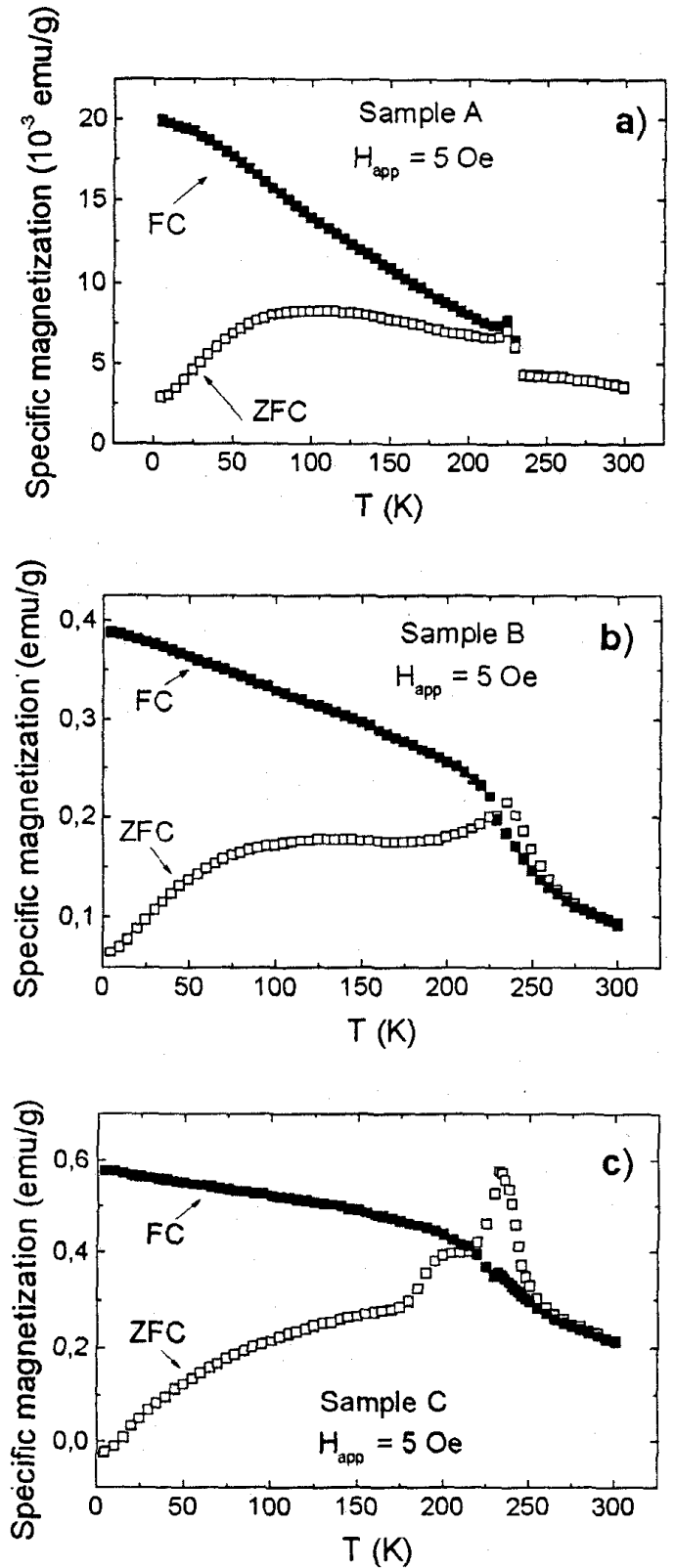

Figure 1. Temperature dependence of the low field magnetization measured in the three samples under $\mathrm{ZFC}$ and $\mathrm{FC}$ conditions

magnetite particles (the $5 \mathrm{~K}$ value of the coercive force decreased with the increase of the concentration of magnetic phase from a value of $298 \mathrm{Oe}$, measured in sample A, down to $272 \mathrm{Oe}$, obtained in sample C) and indistinguishable from zero for temperatures above $175 \mathrm{~K}$. In order to compare our results with the laws $H_{c}(T)=H_{c}(0)\left[1-\left(T / T_{B}\right)^{k}\right]$, describing the coherent rotation prediction for the temperature dependence of the coercive force of uniaxial, non interacting, aligned $(\mathrm{k}=0.5)$ or isotropic $(\mathrm{k}=0.77)$ particles [6] (below the average blocking temperature, $T_{B}$ ) we have plotted the experimental data as a function of $\mathrm{T}^{0.5}$ and of $\mathrm{T}^{0.57}$. As it is possible to observe from Table I, where we present the parameters obtained from the fits to both laws of the low temperature ( $\mathrm{T}<50 \mathrm{~K}$ ) data, that corresponding to oriented particles seems to provide (see the values of the correlation coefficient, r) a better description of the temperature dependence of the coercivity of the samples (nevertheless, none of the two laws are adequate to reproduce our results in the whole considered temperature range). It is also interesting to point out that the blocking temperature values obtained from the fits are independent of the concentration (as one could, in principle, expect from the fact that all the samples were prepared from the same batch of magnetite particles) and clearly lower than the temperatures of the ZFC maxima observed in the low temperature range in samples $A$ and $B$.

TABLE I

FITTING PARAMETERS CORRESPONDING TO THE LAWS DESCRIBING THE TEMPERATURE DEPENDENCE OF THE COERCIVE FORCE IN ORIENTED $(\mathrm{k}=0.5)$ AND ISOTROPIC $(\mathrm{k}=0.77)$ PARTICLES

\begin{tabular}{|c|c|c|c|c|c|c|}
\hline Sample & & $k=0.5$ & & & $k=0.77$ & \\
\hline & $\begin{array}{l}\mathrm{H}_{\mathrm{c}}(0) \\
(\mathrm{Oe})\end{array}$ & $T_{B}(K)$ & $\dddot{r}$ & $\begin{array}{l}H_{c}(0) \\
(\mathrm{Oe})\end{array}$ & $\mathrm{T}_{\mathrm{B}}(\mathrm{K})$ & $r$ \\
\hline A & $442 \pm 12$ & $39 \pm 3$ & 0.9920 & 365 & 33 & 0.9875 \\
\hline B & 436 & 39 & 0.9942 & 362 & 33 & 0.9845 \\
\hline C & 415 & 38 & 0.9920 & 346 & 34 & 0.9832 \\
\hline
\end{tabular}

\section{Discussion}

We associate the low temperature maxima observed in the ZFC curves of the different samples to the blocking of the rotation of the particle magnetic moment with respect to their crystallographic axes (Néel-type relaxation). This idea is supported by the fact that the kerosene is solid at the temperature range of the maxima and therefore able of effectively hindering any particle rotation (Brown-type relaxation). The fact that the temperatures at which these maxima take place are larger, by a factor of the order of 3 , than the blocking temperature estimations obtained from the coercive force fits evidence a large influence of the interparticle (dipolar) interactions on the characteristics of this Neel-type blocking process. Also, according to the coercivity results, the interactions should originate particle aggregates which, considering the large concentration variations and the measurably different low temperature maxima observed in the ZFC curves, should be significantly different in the different samples.

As for the ZFC maxima onseting in all the samples at ca. $225 \mathrm{~K}$, and considering the fact that the kerosene exhibits liquid-like viscosity at temperatures above $230 \mathrm{~K}$ [7], we identify them with the occurrence of Brown relaxation. Since the proportion of blocked particles present, at the temperature range of the maxima, is the largest of all the samples in the most concentrated one (sample $\mathrm{C}$ ) the magnetization change associated to the process is larger in this colloid than in the 

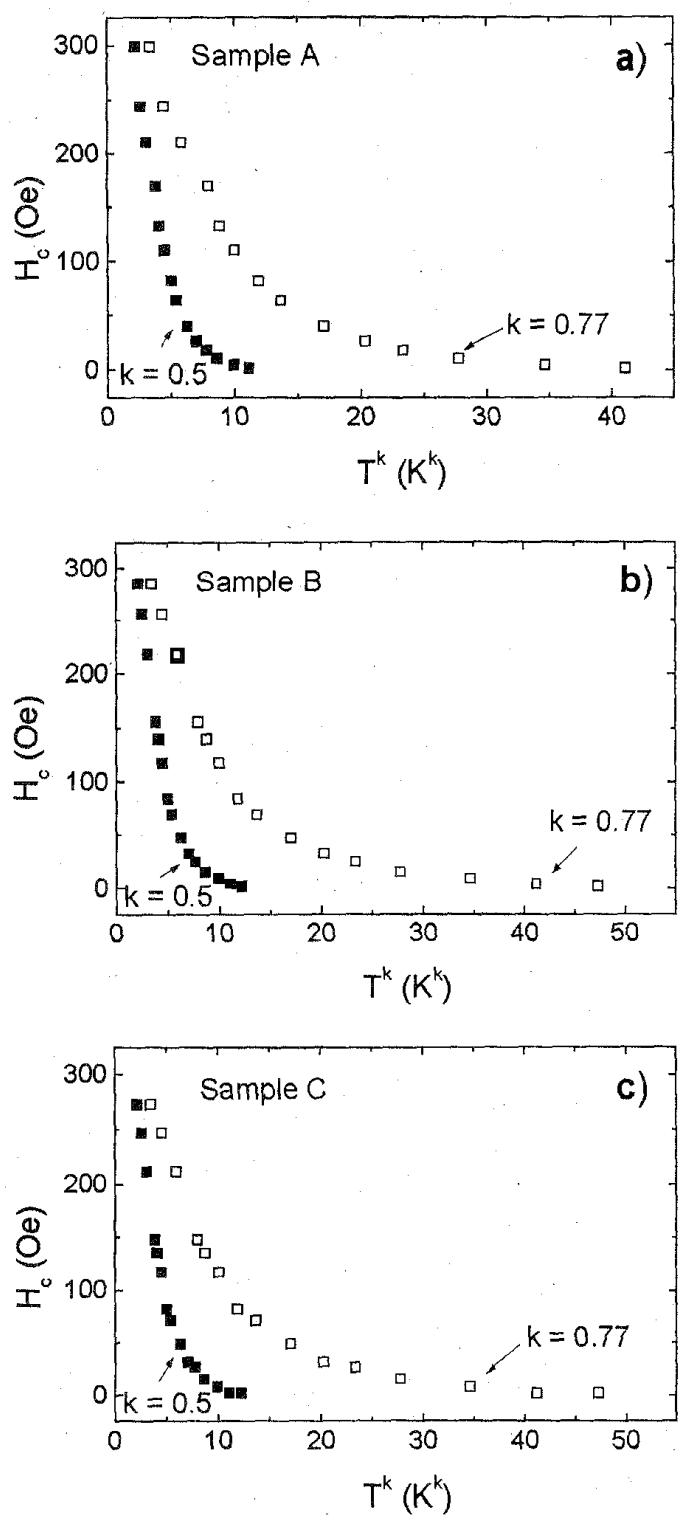

Figure 2. Temperature dependence of the saturation coercivity of our samples plotted as a function of $\mathrm{T}^{0.5}$ (perfectly oriented particle easy axes) and of $\mathrm{T}^{0.77}$ (isotropic easy axes distribution)

others. Finally, we suggest that the intermediate temperature maximum observed in sample $C$ (and the traces of it present in the results corresponding to sample B) could be connected to the simultaneous occurrence of magnetic moment and particle rotations. Again, the corresponding maximum is more pronounced in sample $C$ due to its larger fraction of blocked particles (due to the very different time scale involved in both types of relaxation [8], particle rotation can not take place in unblocked particles).
The temperature dependence of the coercivity is clearly influenced by the well-known Verwey transition (taking place in magnetite at $119 \mathrm{~K}$ and being associated to a change from a high temperature cubic structure to a low temperature monoclinic one) [9]). From the magnetic point of view the Verwey transition, is linked to the development, at low temperatures, of a uniaxial anisotropy. In fact, the coercivity of the samples at temperatures at which the magnetite exhibits cubic structure is negligible whereas it increases rapidly below the transition temperature and, according to our results, behaves, in a limited temperature range, as predicted for uniaxial particles (shape anisotropy could also contributeto this uniaxial character). It is, nevertheless, surprising to obtain a better agreement of our results with the $\mathrm{T}^{0.5}$ law than with that corresponding to isotropically distributed particles. The origin of this should be the occurrence of a large degree of easy axis aligniment of the magnetite particles originated by the dipolar interactions. We should, nevertheless, point out that the nominal average interparticle distances (which decrease from 5.75 diameters in the case of sample A down to 1.25 in that of sample C) are not compatible with the very similar coercivity values measured in the three colloids which again suggests the presence in our colloids of particle aggregates. The presence of aggregates should also influence the reversal mode which could be an incoherent one (fanning). This last point is strongly supported by the comparison of our (approximately concentration independent) experimental coercivity results, which were of the order of $300 \mathrm{Oe}$ at $5 \mathrm{~K}$, with the coercivity value, $\mathrm{H}_{\mathrm{c} C \mathrm{R}}$, corresponding, at this temperature, to the coherent rotation reversal mode that we have evaluated in $8500 \mathrm{Oe}\left(\mathrm{H}_{\mathrm{cCR}}=2 \mathrm{~K}_{\mathrm{aa}} / \mathrm{M}_{\mathrm{S}}\right.$ were $\mathrm{K}_{\mathrm{aa}}(5 \mathrm{~K})$ $=2.1 \times 10^{6} \mathrm{erg} / \mathrm{cm}^{3}$ and $\mathrm{M}_{\mathrm{S}}(5 \mathrm{~K})=6200$ Gauss [10]). The occurrence of an incoherent rotation mode could also be responsible, in addition to the presence of dipolar interactions, for the large differences between the average blocking temperatures obtained from the $\mathrm{ZFC}$ curves and the fit of the coercivity data.

\section{REFERENCES}

[1], S.W. Charles and J. Popplewell, "Ferromagnetic liquids" in Ferromagnetic Materials vol 2, E.P. Wolhfarth Ed., Amsterdam, NorthHolland Publishing Co., 1980, pp. 513-514

[2]. W. Luo, S.R. Nagel, T.F. Rosenbaum and R.E. Rosenweig, "Dipole interactions with Random Anisotropy in a Frozen Ferrofluid" Phys. Rev. Lett. vol. 67, pp. 2721, November 1991.

[3]. K. O'Grady, J. Popplewell and S.W. Charles, "Initial susceptibility of ferrofluids" J. Magn. and Magn. Mater. vol. 39, pp 56, 1983.

[4]. S.E. Khalafalla and G.W. Reimers. IEEE Trans. on Magn. vol. MAG16, pp. 178,1980

[5]. P. Scherrer. Gotting. Nachr., vol. 2.pp. 18, 1918

[6]. H. Pfeiffer. "Relaxation behavior of magnetic particle assemblies due to thermal fluctuations" phys. stat. sol. (a), vol. 20, pp. 233, 1990

[7]. J. Zhang, C. Boyd and W. Luo. "Two mechanisms and a scaling relation for dynamics in ferrofluids" Phys. Rev. Lett. vol. 77, pp. 390, July 1996

[8]. A.M. Konn, P. laurent, P. Talbot and M. Le Floc'h. "Study of relaxation and spin resonance in magnetic liquids". J. Magn. Magn. Mater. vols. 140-144, pp. 367,1995

[9]. S. Krupicka and P. Novak, "Oxide spinels" in Ferromagnetic Materials vol 3, E.P. Wolhfarth Ed., Amsterdam, North-Holland Publishing Co., 1982 , pp. $264-265$

[10]. Landolt and Börnstein "Magnetic oxides and related compounds" part b. Lefever. pp. $65-67$ 